\title{
Lattice Boltzmann Simulation of Permeability and Tortuosity for Flow through Dense Porous Media
}

\author{
Ping Wang \\ Department of Engineering Mechanics, State Key Laboratory for Structural Analysis of Industrial Equipment, \\ Dalian University of Technology, Dalian 116024, China \\ Correspondence should be addressed to Ping Wang; wangp@dlut.edu.cn
}

Received 17 December 2013; Revised 26 May 2014; Accepted 26 May 2014; Published 20 July 2014

Academic Editor: Shaoyong Lai

Copyright (C) 2014 Ping Wang. This is an open access article distributed under the Creative Commons Attribution License, which permits unrestricted use, distribution, and reproduction in any medium, provided the original work is properly cited.

Discrete element method (DEM) is used to produce dense and fixed porous media with rigid mono spheres. Lattice Boltzmann method (LBM) is adopted to simulate the fluid flow in interval of dense spheres. To simulating the same physical problem, the permeability is obtained with different lattice number. We verify that the permeability is irrelevant to the body force and the media length along flow direction. The relationships between permeability, tortuosity and porosity, and sphere radius are researched, and the results are compared with those reported by other authors. The obtained results indicate that LBM is suited to fluid flow simulation of porous media due to its inherent theoretical advantages. The radius of sphere should have ten lattices at least and the media length along flow direction should be more than twenty radii. The force has no effect on the coefficient of permeability with the limitation of slow fluid flow. For mono spheres porous media sample, the relationship of permeability and porosity agrees well with the $\mathrm{K}-\mathrm{C}$ equation, and the tortuosity decreases linearly with increasing porosity.

\section{Introduction}

Fluid flow through porous media has been widespread concern. It plays an important role in the seepage, drainage, and oil engineering. Engineers often need to predict the fluid flow rate of water or oil in porous media. A simple empirical method called Darcy's law is often used [1]; the formula reads

$$
k_{i j}=-\frac{\mu}{p_{, j}}\left\langle v_{i}\right\rangle,
$$

where $v_{i}$ is outflow speed in the direction $i,\langle\rangle$ represents volume average, $\mu$ is the viscosity coefficient of the fluid, $p_{j}$ is the pressure gradient in direction $j$, and $k_{i j}$ is the permeability matrix. Permeability matrix is assumed to be diagonal and positive definite. The elements of permeability matrix can be measured by experimental methods or estimated by computational methods.

Many factors affect permeability, such as porous media structure, fluid physical properties, and degree of fluid saturation. This paper studies the impact of porous media structure only. The traditional predictive research is usually concerned with the relationship between permeability and porosity. In addition to that, specific surface of the particle, shape of the particle, and tortuosity also have a significant impact on permeability. But effects of these factors are hard or even impossible to measure accurately; these problems need pore-scale simulation of fluid flow in porous media.

Because of the complexity of fluid flow in porous media, except for some very simple question, it is difficult to get the analytical solution. With the development of the porous media geometry visualization, computing power, and computational methods, it is possible to simulate directly fluid flow in pore-scale porous media.

The LBM method has been applied to this field since it was introduced in 1989. Ghassemi and Pak [2] studied the effects of permeability and tortuosity on flow through particulate media by LBM and DEM; the relationships between permeability and tortuosity with other parameters such as particles diameter, grain specific surface, and porosity were identified. The results show that the relationship between permeability and porosity of the porous media agreed the K-C formula well, but there is a bit deviation in the high porosity region; 
tortuosity almost linearly decreases with the porosity, and the particle diameter does not affect tortuosity any longer after it becomes large enough.

Sun et al. [3] introduced a multiscale method. Microstructural attributes such as occluded/connected porosity and geometrical tortuosity are extracted using new computational techniques to form digital images of porous media, and a LBM/FEM scheme is used to obtain homogenized effective permeability at specimen scale. The results show that the permeability depends not only on the porosity, but also on the connectivity and the tortuosity.

Experimental measurements and LBM simulation on samples of packed particle beds were researched by Videla et al. [4]. Due to the resolution limit, when the particle diameter is greater than 500 microns, the experimental results and numerical results are consistent. The impact of the relaxation time on permeability was also studied. Vidal et al. [5] verified that the LBM numerical permeability of the porous media of spherical particles agrees with experimental data very well. When the size distribution of spherical particles disperses to a certain extent, the results start to deviate from $\mathrm{K}-\mathrm{C}$ formula, and a modified formula was given according to the results. Jeong [6] studied the micro flows through granular porous media at various Knudsen numbers, the correlation between the permeability, the porosity, and the Knudsen number is derived, a new fitting formula is given, and the effect of rarefaction on the permeability is also discussed. van der Hoef et al. [7] simulated fluid flow past mono- and bidisperse random arrays of spheres with LBM, the calculated permeability is consistent with experimental results, and a new drag force formula is given. The effect of the arrangement of the particles and particle shape on permeability anisotropy was researched by Stewart et al. [8].

In this work, the permeability and tortuosity of fluid flow in three-dimensional dense porous media have been simulated and analyzed. The effects of porosity and the size of particle have been studied. The DEM is described in Section 2. Numerical model of LBM is present in Section 3. Calculation results are given in Section 4 and the Conclusion in Section 5.

\section{Discrete Element Method and Porous Media Generation}

2.1. Discrete Element Method (DEM). DEM is an explicit numerical method for modeling motion of distinct particles. Since this method was introduced by Cundall and Strack [9], it has become a powerful tool and has been used to research a wide range of problems in science and engineering.

In DEM, the granular porous media are regarded as an assembly of distinct rigid particles. When overlap occurs between particles (or particle and wall), the contact force separates them from each other. Using the force-displacement law, the force system of each particle can be summed. The displacement and velocity of each particle can be calculated by Newton's second law of motion.
The force-displacement law gives the relationship between the contact force and the overlap between particles (or particle and wall) by the following equations:

$$
\Delta F^{n}=K^{n} \Delta^{n}, \quad \Delta F^{\tau}=K^{\tau} \Delta^{\tau},
$$

where $\Delta F, K$, and $\Delta$ are increments of force, stiffness, and overlap between particles, respectively. The superscripts $n$ and $\tau$ represent normal and tangential direction of overlap plane.

The contact forces consist of normal force and tangential force. The tangential force is related to the normal force by the Coulomb frictional law. If $F_{p, i}^{\tau} \leq \mu_{s} F_{p, i}^{n}$, relative translation motion will not occur. Here $\mu_{s}$ is the sliding friction coefficient between the contact particles, and $F_{p, i}^{\tau}, F_{p, i}^{n}$ are the normal and tangential forces on particle $p$ in position $i$.

Newton's second law of motion describes the relationship between the acting forces and movement of particle. For sphere $p$, the law reads

$$
\begin{aligned}
& m_{p} \ddot{x}_{p, i}=\sum\left(F_{p, i}^{\tau}+F_{p, i}^{n}\right)-F_{p, i}^{d}, \\
& I_{p} \ddot{\theta}_{p, i}=\sum\left[\left(\mathbf{F}_{p}^{\tau} \times \mathbf{R}_{p}\right)_{i}\right]-M_{p, i}^{d},
\end{aligned}
$$

where $m_{p}, I_{p}$ are the mass and inertia of sphere particle $p$; $\ddot{x}_{p, i}$ and $\ddot{\theta}_{p, i}$ are linear and angular accelerations in direction $i ; F_{p, i}^{d}, M_{p, i}^{d}$ are the global damp force and moment; $\mathbf{R}_{p}$ is the vector from the center of sphere to the contact point.

2.2. Porous Media Generation Using DEM. The first step of modeling is producing dense assembly of granular particles. We retrieve it with opening LAMMPS code. The details are shown in Figures 1 and 2. A domain whose size is $100 \times$ $100 \times 1000$ by lattice unit is created first. Periodic boundary condition is used in the two horizontal directions. In vertical direction, rigid and impassable planes are set at the top and bottom boundaries.

The rigid particles are created in a small region near the top boundary first, in order to reduce the probability of overlap; the ratio of particles volume to domain is set to ten percent. The position of each particle will be determined when it does not overlap with any other particles. Because the percentage of particles is low, this process is easy to implement.

After the loose assembly is created in the specific region, it starts to fall down under gravity (Figure 1). When the particles leave the initial region totally, new particles will be created. This process is repeated until enough particles appear in computational domain. The particles interact with other particles, and the dense assembly of particles forms under gravity at last.

To get loose porous media with a desired porosity, the radii of particles are shortened with same scale, but the amount and positions of particles are unchanged. 


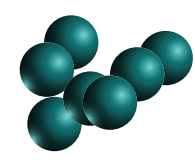

$$
\sqrt{\text { Gravity }}
$$

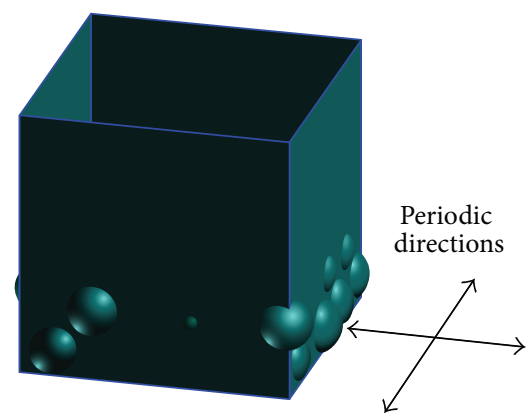

FIGURE 1: Generation of dense porous media.

\section{Lattice Boltzmann Method and Simulation of Fluid Flow}

3.1. LBM. The simplest LBM model is known as the BGKBoltzmann model. It was first introduced by Bhatnagar et al. [10]. The evolutional equation reads

$$
f_{\alpha}\left(\mathbf{r}+\mathbf{e}_{\alpha} \delta t, t+\delta t\right)-f_{\alpha}(\mathbf{r}, t)=-\frac{1}{\tau}\left[f_{\alpha}(\mathbf{r}, t)-f_{\alpha}^{e q}(\mathbf{r}, t)\right],
$$

where $f_{\alpha}, \mathbf{r}, t$, and $\mathbf{e}_{\alpha}$ are probability distribution function of particle in direction $\alpha$, space position, time, and microscopic velocity, respectively. $f_{\alpha}$ moves from $\mathbf{r}$ to $\mathbf{r}+\mathbf{e}_{\alpha}$ in a time step. The right term indicates the colloids function, that is, relaxation function. Probability distribution function of particle approaches $f_{\alpha}^{e q}(\mathbf{r}, t)$ (the local equilibrium distribution function). $f_{\alpha}^{e q}(\mathbf{r}, t)$ is the function of local density and velocity. The relaxation factor $\tau$ indicates relaxation speed rate and is related to fluid viscosity coefficient by $\tau=3 \nu+$ $0.5>0.5$. Every evolution procedure consists of two stages, a propagation stage and a collision stage. Under limitation of low fluid flow, the steady solution of (4) is the solution of Navier-Stokes equations.

The final fluid flow state is evidently influenced by boundary conditions. The interface of the particles and fluid is assumed as a no-slip boundary. The simplest method which is called half-way bounce-back method is adopted here. The pressure drop is implemented by placing a body force on fluid. Periodic boundary condition is used in all directions. The D3Q19 model is used to calculate the fluid flow among the interval of porous media (Figure 3 ). This model includes a stationary part and eighteen moving parts. They are $\left\{\mathbf{e}_{\alpha}\right\}=E^{T}\{\mathbf{i}, \mathbf{j}, \mathbf{k}\}^{T}$; here $\left\{\mathbf{e}_{\alpha}\right\}=\left\{\mathbf{e}_{0}, \mathbf{e}_{1}, \mathbf{e}_{2}, \ldots, \mathbf{e}_{18}\right\}$ and $E=\left\{\begin{array}{ccccccccccccccccccc}0 & 1 & -1 & 0 & 0 & 0 & 0 & 1 & -1 & 1 & -1 & 1 & -1 & 1 & -1 & 0 & 0 & 0 & 0 \\ 0 & 0 & 0 & 1 & -1 & 0 & 0 & 1 & -1 & -1 & 1 & 0 & 0 & 0 & 0 & 1 & -1 & 1 & -1 \\ 0 & 0 & 0 & 0 & 0 & 1 & -1 & 0 & 0 & 0 & 0 & 1 & -1 & -1 & 1 & 1 & -1 & -1 & 1\end{array}\right\}$.
Local equilibrium distribution function is given by

$$
f_{\alpha}^{e q}=\rho w_{\alpha}\left(1+\frac{\mathbf{e}_{\alpha} \cdot \mathbf{u}}{c_{s}^{2}}+\frac{\left(\mathbf{e}_{\alpha} \cdot \mathbf{u}\right)^{2}}{2 c_{s}^{4}}-\frac{u^{2}}{2 c_{s}^{2}}\right),
$$

where $\rho, c_{s}, \mathbf{u}$, and $w_{\alpha}$ are density, sound velocity, velocity vector, and constant coefficient, respectively. The sound velocity equals $\sqrt{3} / 3$ and the constant coefficients are

$$
w_{i}= \begin{cases}\frac{1}{3}, & i=0 \\ \frac{1}{18}, & i=1-6 \\ \frac{1}{36}, & i=7-18\end{cases}
$$

New density $\rho(\mathbf{r}, t)$ and velocity $\mathbf{v}(\mathbf{r}, t)$ of fluid can be extracted from probability distribution function of particle by equations

$$
\sum_{\alpha} f_{\alpha}=\rho, \quad \sum_{\alpha} f_{\alpha} \mathbf{e}_{\alpha}=\rho \mathbf{u}
$$

3.2. Simulation of Fluid Flow Using LBM. As input condition, assembly of dense particles created by DEM is transmitted into LBM code. Lattice is signed by TRUE if it does not locate inside any particle, FAULSE if it does. All particles are fixed during simulation procedure, and they interact with fluid by bounce-back boundary condition [11].

Lattice should be fine enough to catch the details of the particles. We place ten lattices along the radius of sphere in our simulation. The computer load is quite big, and an elementary lattice structure is $240 \times 100 \times 100$.

In flow direction, additional layers are added to the ends of domain. The path of fluid flow is shown in Figure 4. Periodic boundary condition is used in the other two directions.

A uniform body force drives fluid to flow; the GZS model is adopted in our simulation [11]. All the simulations start from a zero velocity filed; convergence to the steady solution is examined by the following criterion:

$$
\text { error }=\frac{\sum\left(\left|\mathbf{v}^{t+1}-\mathbf{v}^{t}\right|\right)}{\sum\left|\mathbf{v}^{t}\right|}<10^{-6}
$$

The summation covers all the fluid lattice sites, and superscript denotes the time steps.

\section{Results and Discussions}

The permeability of the porous media can be computed by formula (1) from steady flow of fluid. The tortuosity is defined by $T=\sum|\mathbf{v}(\mathbf{r})| / \sum\left|v_{i}(\mathbf{r})\right|$, where $\mathbf{v}(\mathbf{r})$ and $v_{i}(\mathbf{r})$ are the velocity vector and the velocity component in flow direction $i$ at position $\vec{r}$, respectively.

Permeability and Lattice Number of the Radii. The space is dispersed into regular lattice point in the LBM. The porous media particles are described by the lattice point. In order to 


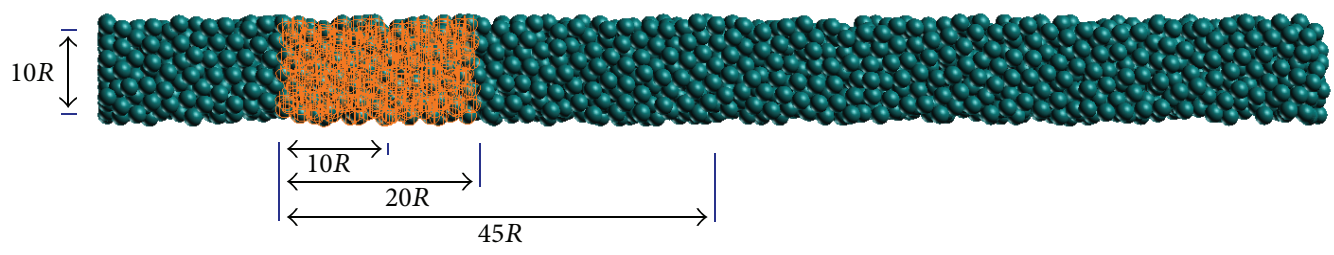

Figure 2: Computational domain and the whole sample.

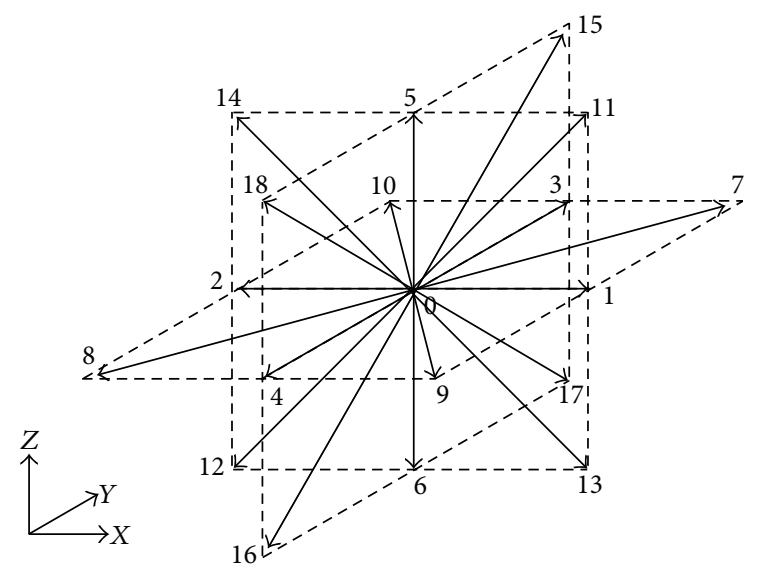

FIgure 3: Particle distribution function on a D3Q19 lattice.

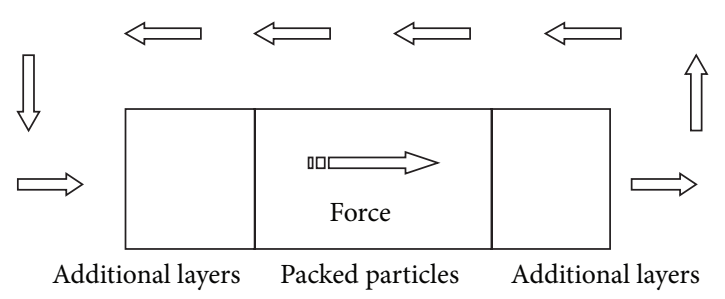

FIGURE 4: Sketch of fluid flow path.

accurately capture the shape of the particles, enough lattices must be placed along the radius of the particle. The more the lattices are, the more accurate the particles are depicted, but it also brings the problem of excessive calculation. Therefore, we need to find a balance point between accuracy and computation burden.

In this paper, the lattice unit is applied always. To simulating the same physical problem, the key criteria numbers of simulations and real physical problem should be matched. These key criteria numbers are reference length $L_{r}$, reference density $\rho_{r}$, and reference velocity $u_{r}$. They are read

$$
L_{r}=\frac{L^{\prime}}{L}, \quad \rho_{r}=\frac{\rho^{\prime}}{\rho}, \quad u_{r}=\frac{c_{s}^{\prime}}{c_{s}}, \quad L_{r} u_{r}=\frac{v^{\prime}}{\nu},
$$

here $L, \rho, t$, and $v$ are length, density, time, and viscosity in lattice unit respectively, and $L^{\prime}, \rho^{\prime}, t^{\prime}, v^{\prime}$, and $c_{s}^{\prime}$ are correspond parameters in physical unit respectively.

Therefore, the viscosity and body force must be adjusted simultaneously. The permeability is obtained with different lattice number. Figure 5 shows the relationship between permeability and the number of lattices among the radius; the permeability enlarges with increase of lattice number. For controlling the computational time (considering machine memory and convergence time), ten lattices are placed along the radial direction (red data points). According to Videla et al.s [4] work, the difference between experimental measurements and numerical simulations is less than $30 \%$ when the ratio of diameter to resolution is bigger than 25 . If the ratio is less than 20 (about 17.5 and 12.5), the difference is very big. Our numerical results are consistent with Videla's conclusion.

Permeability and Media Length. Along the flow direction, the sample of porous media should guarantee enough length. The permeability of the media should be independent of media length in the flow direction. Fluid flow in porous media for different lengths of sample is simulated (see Figure 2); Figure 6 shows the variations of permeability versus the media length in the flow direction. From the figure, when the ratio of channel length to radius is more than 20 , variation of permeability is very small. This media length is used in all our simulations (red data points).

Permeability and Body Force. Similarly, the permeability of porous media should not depend on the body force (pressure gradient). Figure 7 depicts the permeability results of the same sample under different body force. The permeability remains unchanged when the body force is small; the permeability is significantly increasing when the body force becomes larger. It should be noted that maximum speed is 0.0124 when the body force is $3.1623 e-4$. In the following calculation, the body force is taken as $1 e-5$ (the red data point in Figure 7).

Permeability and Porosity. About relationship between porosity (void ratio) and permeability, researchers proposed some different equations. Carman introduced and extended the K$\mathrm{C}$ equation [12]; the equation reads

$$
k=9.81 \times 10^{4} \frac{1}{S_{0}^{2} C_{C-K}} \frac{e^{3}}{1+e}=C_{1} \frac{e^{3}}{1+e} .
$$

$C_{1}$ is a constant parameter which is related to the specific surface area, tortuosity, and fluid physics, and $e=\varepsilon /(1-\varepsilon)$ is void ratio. Ghassemi's simulation gave $k=C_{2}\left(e^{4.2} /(1+e)\right)[2]$. In order to study the influence of porosity on permeability, a series of porous media samples with uniform sphere particles is simulated by LBM. All the samples are made of sphere particles with the same diameter, but they are different in 


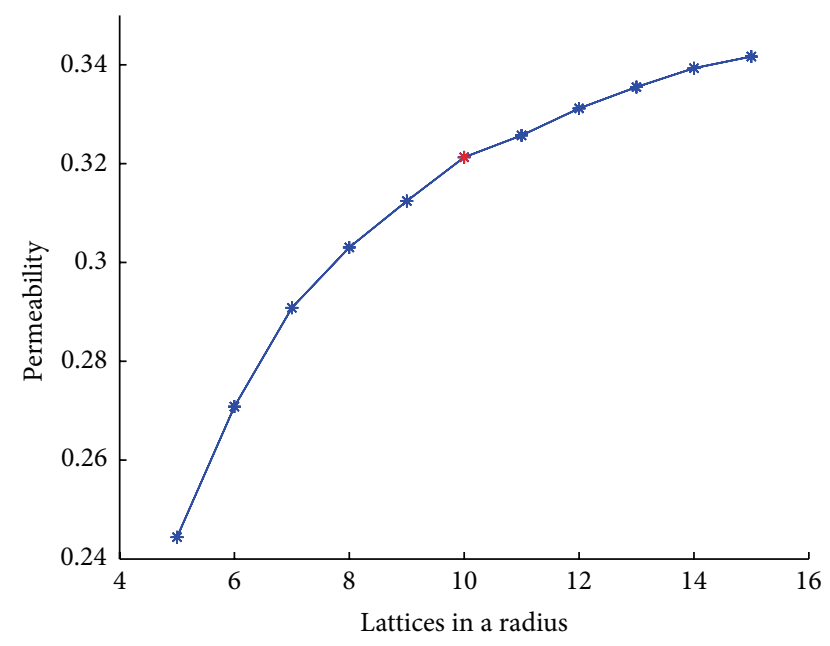

FIGURE 5: Variations of permeability versus lattices along radius.

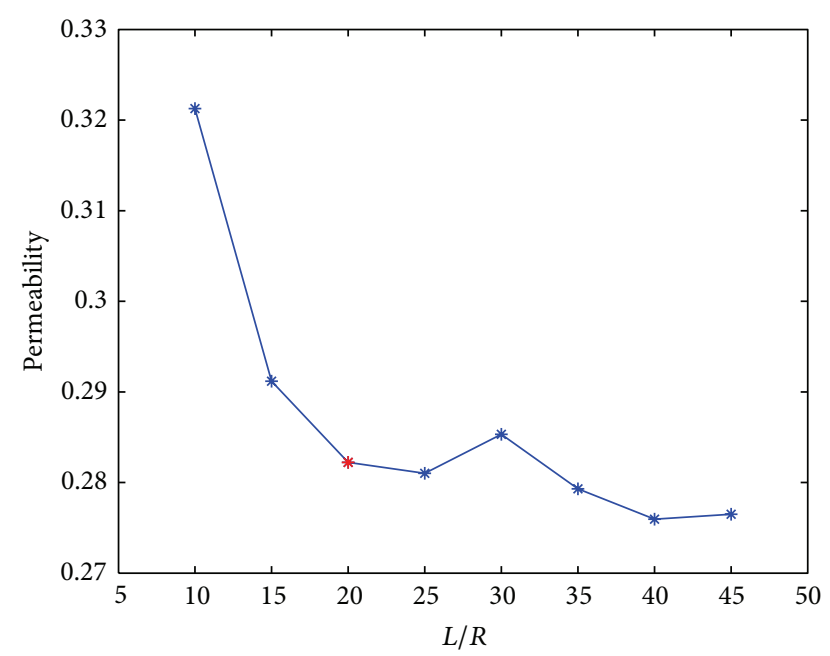

FIGURE 6: Variations of permeability versus length.

porosity. In Figure 8 , the relationship between regularization of the permeability $k^{\prime}=k / C$ and void radio is shown. The curve fits well with the $\mathrm{K}-\mathrm{C}$ equation. Using least squares method, we have $k=C_{3}\left(e^{3} /(1+e)\right)=1.8424\left(e^{3} /(1+\right.$ $e)$ ). When the void ratio is less than 1 , the three results are similar; for large porosity, our numerical results are also consistent with K-C equation, but are different from Ghassemi's results. Ghassemi's simulation is based on twodimensional porous media, and his model cannot capture all the physical characteristics of real porous media; the author elaborated this fact in his paper.

Permeability and Particle Size. Figure 9 depicts the relationship between permeability and particle size; the porosity remains constant $(\varepsilon=0.5)$ in all samples. According to the $\mathrm{K}-\mathrm{C}$ equation, the permeability is inversely proportional to the square of the specific surface area. For the homogeneous sphere porous media, the permeability should be proportional to the square of the diameter. From our results, the

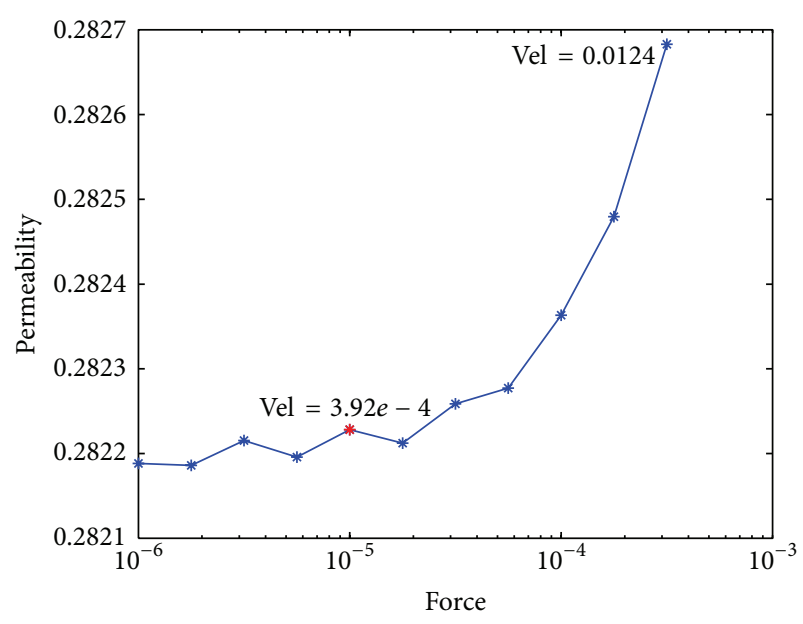

FIGURE 7: Variations of permeability versus body force.

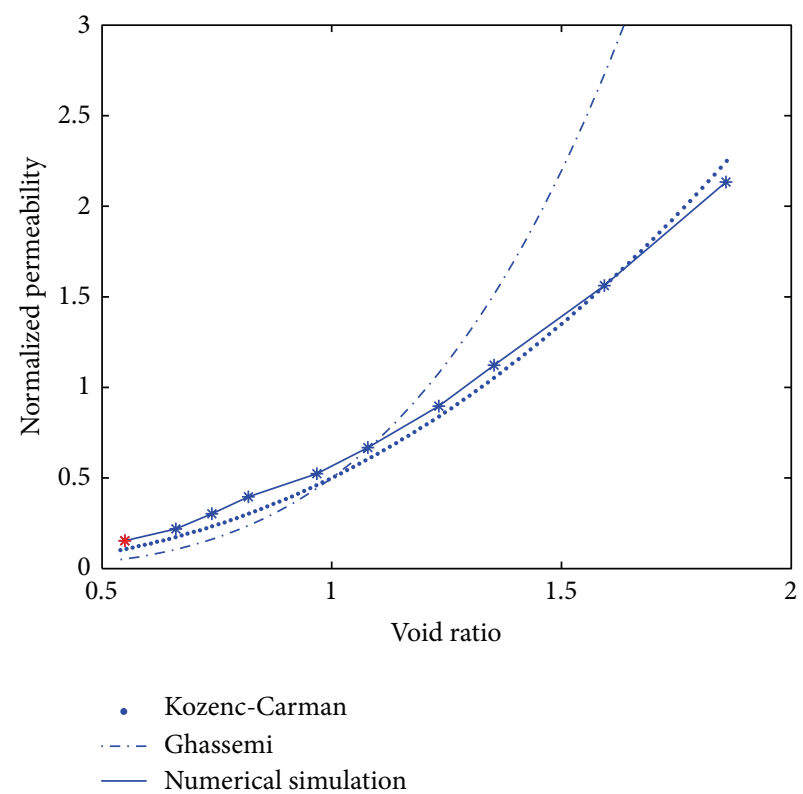

FIGURE 8: Variations of permeability versus porosity.

relationship between the permeability and the radius does not seem to comply with this rule. In fact, it is because the range of particle radius is narrow and the permeability is basically proportional to the radius.

Tortuosity and Porosity. For the relationship between tortuosity and porosity, different researchers have given different results also. Koponen and Ghassemi's results show that the tortuosity decreases linearly when porosity increases [13]. Yet, Boudreau gave the nonlinear relationship [14]; Dias believes that exponential relationship is better [15]. For porous media of uniform particles, Figure 10 depicts the relationship between tortuosity and porosity. The tortuosity essentially decreases linearly with increasing porosity. But our result is much lower than the absolute value of Koponen and Ghassemi's results. The reason is that the dead end will 


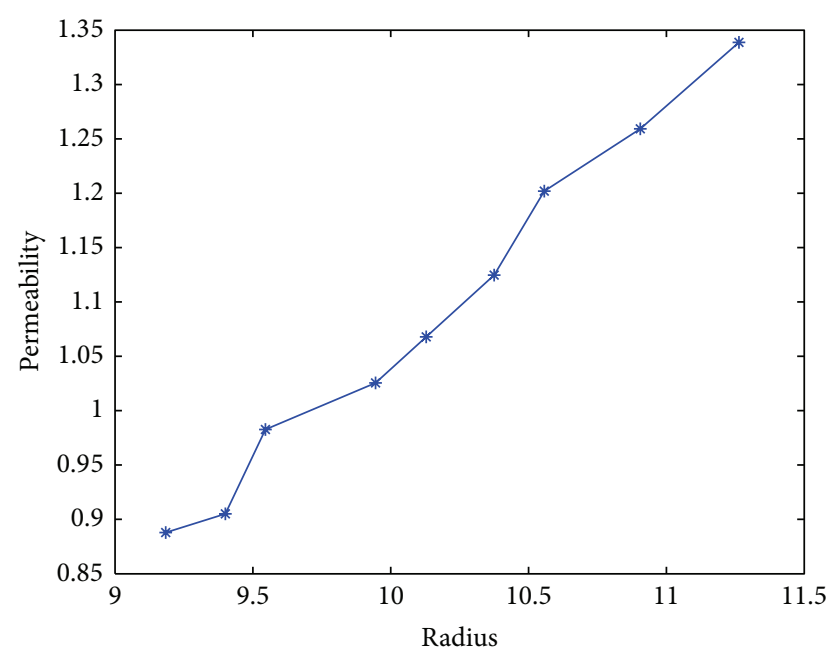

FIGURE 9: Variations of permeability versus particle radius.

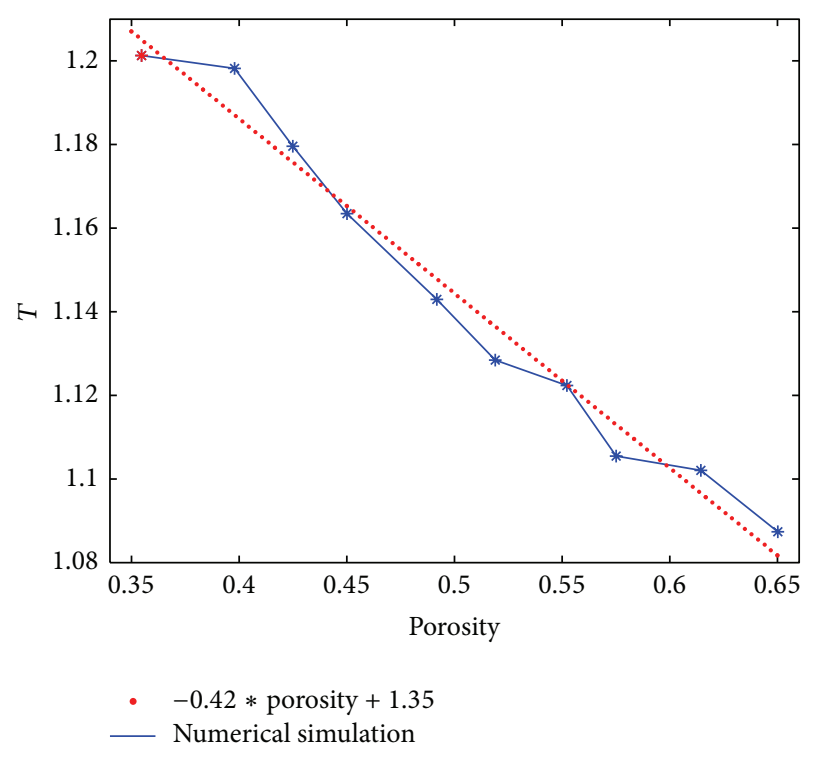

FIGURE 10: Variations of tortuosity versus porosity.

not form in the three-dimensional porous media samples. For example, when the porosity is equal to 0.5, Koponen and of Ghassemi's results are 1.4 and about 1.33, respectively; our result is 1.14 . For the slope of the curve, the results of our calculations (about -0.42) and Ghassemi (-0.45) are relatively close and far from Koponen's result $(-0.8)$. The porous media in Koponen's paper are composed by small rectangles. The channel among porous media has more twists and turns, and the permeability is more sensitive to the porosity. Therefore, when the porosity increases, the tortuosity decreases faster.

\section{Conclusion}

DEM and LBM are used to simulate the fluid flow in the dense porous media; the ability of LBM to resolve this problem is confirmed. The relationship between permeability, tortuosity and porosity, and particle size is researched. In our calculation, all the sample particles are uniform size, rigid, and fixed; high porosity samples are created through rescaling of dense samples. We obtain the following conclusions.

LBM is suitable for the simulation of flow in porous media because of its inherent advantages. The results show that the radius of the sphere should not be less than ten lattices; the ratio of channel length to radius should be larger than 20. Under the limitation of low speed, body force has little influence on the calculation results. If relaxation time is equal to one, the convergence can be achieved quickly, or the convergence is extremely slow. For porous media with uniform spheres, the relationship of permeability and porosity basically meets the K-C formula, and the tortuosity decreases linearly with increasing porosity.

Simulation of samples with a wide range of particle size will be run in further work, and the permeability calculation of nonuniform and nonspherical particle samples will be studied also.

\section{Conflict of Interests}

The author declares that there is no conflict of interests regarding the publication of this paper.

\section{Acknowledgments}

The project was supported by the National Natural Science Foundation (11102034) and the Important National Science \& Technology Specific Project (2011ZX02403-004).

\section{References}

[1] H. Darcy, Determination des Lois d'Ecoulement de l'eau a Travers le Sable, Les Fontaines Publiques de la Ville de Dijon, Victor Dalmont, Paris, France, 1856.

[2] A. Ghassemi and A. Pak, "Pore scale study of permeability and tortuosity for flow through particulate media using Lattice Boltzmann method," International Journal for Numerical and Analytical Methods in Geomechanics, vol. 35, no. 8, pp. 886-901, 2011.

[3] W. C. Sun, J. E. Andrade, and J. W. Rudnicki, "Multiscale method for characterization of porous microstructures and their impact on macroscopic effective permeability," International Journal for Numerical Methods in Engineering, vol. 88, no. 12, pp. 1260-1279, 2011.

[4] A. R. Videla, C. L. Lin, and J. D. Miller, "Simulation of saturated fluid flow in packed particle beds-the lattice-Boltzmaan method for the calculation of permeability from XMT images," Journal of the Chinese Institute of Chemical Engineers, vol. 39, no. 2, pp. 117-128, 2008.

[5] D. Vidal, C. Ridgway, G. Pianet, J. Schoelkopf, R. Roy, and F. Bertrand, "Effect of particle size distribution and packing compression on fluid permeability as predicted by latticeBoltzmann simulations," Computers and Chemical Engineering, vol. 33, no. 1, pp. 256-266, 2009.

[6] N. Jeong, "Advanced study about the permeability for microporous structures using the lattice Boltzmann method," Transport in Porous Media, vol. 83, no. 2, pp. 271-288, 2010. 
[7] M. A. van der Hoef, R. Beetstra, and J. A. M. Kuipers, "Lattice-Boltzmann simulations of low-Reynolds-number flow past mono- and bidisperse arrays of spheres: results for the permeability and drag force," Journal of Fluid Mechanics, vol. 528, pp. 233-254, 2005.

[8] M. L. Stewart, A. L. Ward, and D. R. Rector, "A study of pore geometry effects on anisotropy in hydraulic permeability using the lattice-Boltzmann method," Advances in Water Resources, vol. 29, no. 9, pp. 1328-1340, 2006.

[9] P. A. Cundall and O. D. L. Strack, "The distinct numerical model for granular assemblies," Geotechnique, vol. 29, no. 1, pp. 47-65, 1979.

[10] P. L. Bhatnagar, E. P. Gross, and M. Krook, "A model for collision processes in gases. I. Small amplitude processes in charged and neutral one-component systems," Physical Review, vol. 94, no. 3, pp. 511-525, 1954.

[11] Z. L. Guo and C. G. Zheng, Theory and Applications of Lattice Boltzmann Method, Science Press, Beijing, China, 2009.

[12] P. C. Carman, "Fluid flow through granular beds," Transactions of the Institution of Chemical Engineers, vol. 15, pp. 150-166, 1937.

[13] A. Koponen, M. Kataja, and J. Timonen, "Permeability and effective porosity of porous media," Physical Review E: Statistical Physics, Plasmas, Fluids, and Related Interdisciplinary Topics, vol. 56, no. 3, pp. 3319-3325, 1997.

[14] B. P. Boudreau, "The diffusive tortuosity of fine-grained unlithified sediments," Geochimica et Cosmochimica Acta, vol. 60, no. 16, pp. 3139-3142, 1996.

[15] R. Dias, J. A. Teixeira, M. Mota, and A. Yelshin, "Tortuosity variation in a low density binary particulate bed," Separation and Purification Technology, vol. 51, no. 2, pp. 180-184, 2006. 


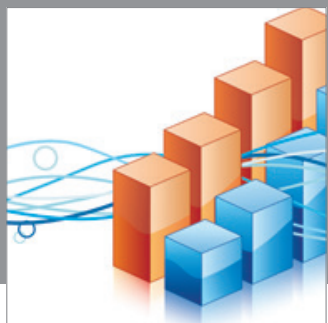

Advances in

Operations Research

mansans

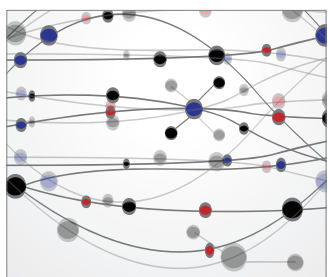

The Scientific World Journal
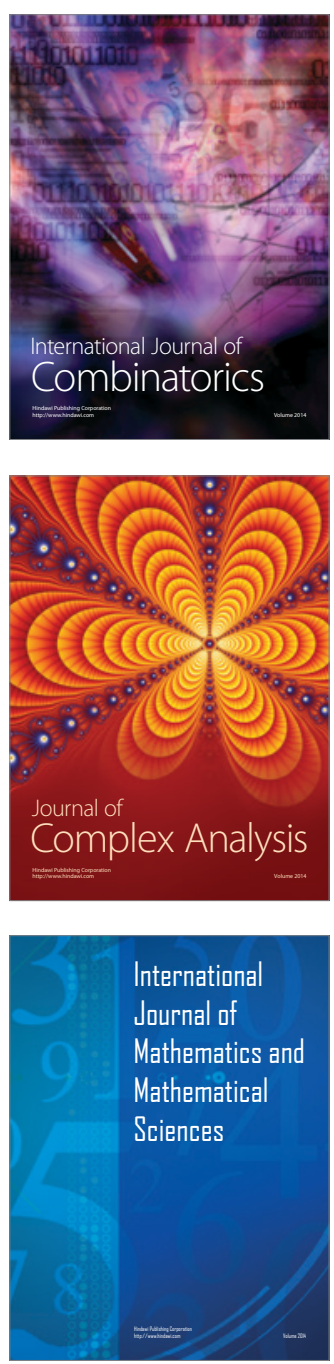
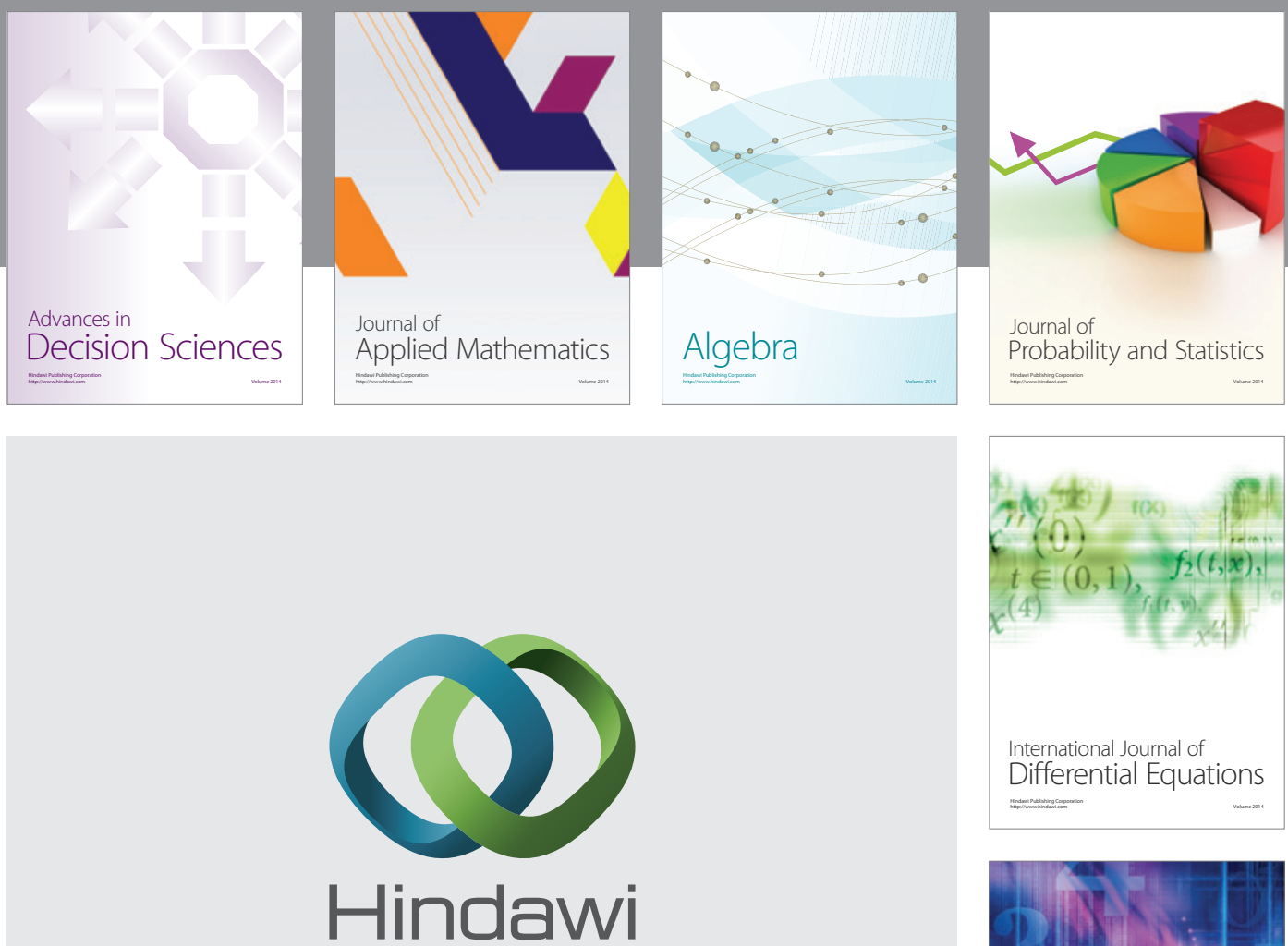

Submit your manuscripts at http://www.hindawi.com
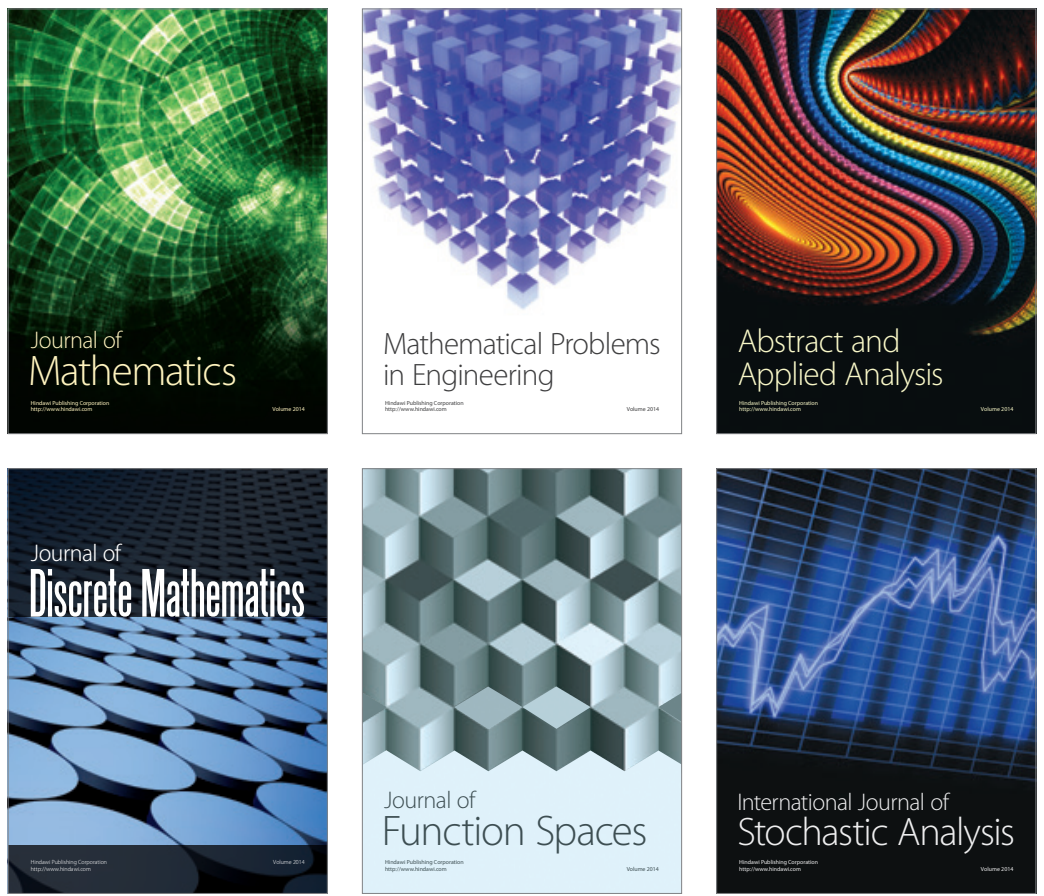

Journal of

Function Spaces

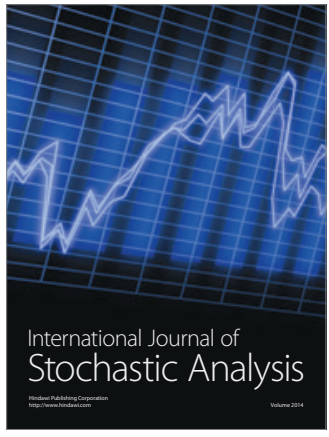

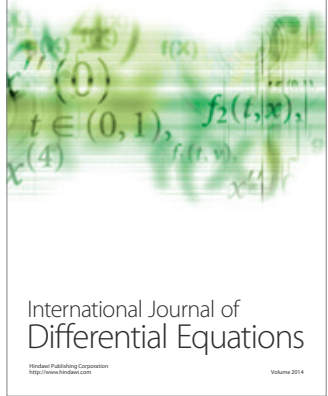
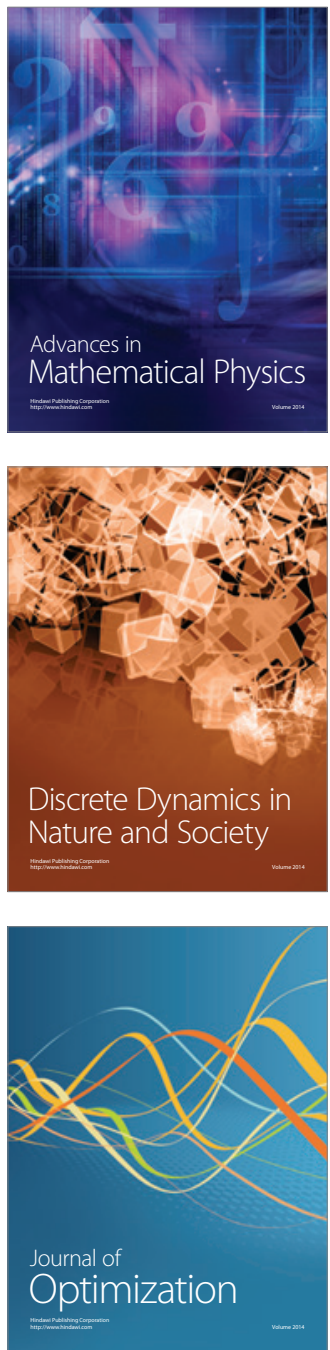